\title{
Assessment of Physico-chemical Properties in Some Soils of District Baramulla under Different Land Use Systems
}

\author{
Sumaira Shafi*, Mushtaq A. Wani and Shaista Nazir
}

Division of Soil Science and Agricultural Chemistry, SKUAST-K (India)

*Corresponding author

\section{A B S T R A C T}

\section{Keywords}

Soil, Physicochemical properties, Land uses

Article Info

Accepted:

10 November 2018

Available Online:

10 December 2018
The experiment was carried out at, Faculty of Agriculture, Wadura-Sopore, SKUAST-K during 2017-2018, to study "Soil Physico-chemical properties in some soils of district Baramulla under different Land use systems". The land use systems studied included apple, paddy, maize, vegetables, forest and pasture. Composite soil samples were collected randomly for each land use system at a depth $(0-20 \mathrm{~cm})$ and were analyzed for soil texture, $\mathrm{pH}, \mathrm{EC}$, $\mathrm{CEC}$, organic carbon and calcium carbonate. The soils were found to possess medium to moderately fine texture.

\section{Introduction}

Land use is characterized by the arrangements, activities and inputs people undertake in a certain land cover type to produce, change or maintain it (Abad et al., 2014). Land use systems significantly affected the clay, the silt and the sand fractions and affect the distribution and supply of soil nutrients by directly altering soil properties like exchangeable basic and acidic cations (Stutter et al., 2004). Land use and soil management practices influence the soil nutrients and related soil processes, such as erosion, oxidation, mineralization, and leaching, etc (Celik, 2005; Liu et al., 2010). As a result, it can modify the processes of transport and redistribution of nutrients. The supply of nutrients to the plants in an appropriate quantity at the correct time is essential for sustainable yield. Soil organic matter, crop residues and manure plays a vital role in supplying nutrients to plants. The nutrient dynamics forms the basis for fertilizer application to get regular and optimum yield without losing the soil health. An appropriate land use system plays an important role in improving soil quality through the addition of leaf litter, binding of soil through root system, checking runoff, soil and nutrient losses, etc. A systematic characterization of soil is of prime importance for evolving suitable agronomic practices and predicting their ability in relation to the different land use systems. Therefore, the present study entitled "Assessment of physico-chemical properties 
in some soils of district Baramulla under different Land use systems" has been carried out.

\section{Materials and Methods}

The present investigation entitled, "Assessment of physico-chemical properties in some soils of district Baramulla under different Land use systems", was carried out at, Faculty of Agriculture, Wadura - Sopore, SKUAST-K during 2017-18 (Fig. 1). The details of the techniques followed and materials used during the course of this investigation are presented as;

For investigating the "Assessment of soil physico-chemical properties in some soils of District Baramulla under different Land use systems", composite surface soil samples (0$20 \mathrm{~cm}$ ) were collected from the following locations in district Baramulla. The exact locations of these sites are presented in Table 1.

No. of land use systems used: $\quad 05$

Horticulture: Fruits- apple

Agriculture (Irrigated - paddy)

Agri-horti (Maize \& Vegetables)

Forestry

Pasture

\section{Design of survey}

No. of locations per land use system: 03

No. of sites per location:

03

No. of replications per site:

Design: Stratified Random Sampling

\section{Preparation of soil samples}

The soil samples were air dried in shade, ground with wooden mallet and passed through a $2 \mathrm{~mm}$ plastic sieve. For organic carbon determination, the soil was passed through $0.2 \mathrm{~mm}$ sieve. After mixing thoroughly, the processed soil samples were stored in polythene bags for laboratory studies.

Physico-chemical characteristics of soil

Mechanical analysis (Particle size distribution)

The mechanical analysis of the soil samples was done by following the International Pipette method as described by Piper (1966).

Soil reaction $(\mathbf{p H})$ : Soil $\mathrm{pH}$ was determined in 1:2.5 soil: water suspension with digital glass electrode pH meter (Jackson, 1973).

Electrical conductivity (EC): Electrical conductivity of the suspension liquid of 1: 2.5 soil: water suspension was determined with the help of Solu bridge conductivity meter at $25^{\circ} \mathrm{C}$ (Jackson, 1967).

Cation Exchange Capacity (CEC): Cation exchange capacity of soil was determined according to the procedure outlined by Rhoades (1982).

Calcium Carbonate $\left(\mathrm{CaCO}_{3}\right)$ : Calcium carbonate was determined by the method given by Puri (1930).

Organic Carbon (OC): Organic carbon was determined by Walkley and Black's rapid titration method.

\section{Results and Discussion}

\section{Physico-chemical properties of soil}

\section{Particle size distribution (Soil texture)}

Particle size distribution of the soil is a governing factor for assessing nutrient supplying power, aeration and drainage of soils. The data presented in Table 2, with regard to particle size distribution indicated varied mechanical make up in district Baramulla. The course sand fraction in surface 
soils varied from 0.17 to $3.60,1.20$ to 10.56 , 1.30 to $2.88,1.10$ to $3.20,1.20$ to 6.30 and 0.16 to 8.10 per cent with the mean values of $2.48,4.26,2.01,2.18,3.06$ and 2.78 per cent in apple, paddy, maize, vegetables, forest and pasture respectively.

The fine sand fraction varied from 15.00 to $44.45,16.62$ to $32.21,17.10$ to $19.90,16.10$ to $37.31,20.68$ to 54.50 and 22.00 to 51.89 per cent with the mean values of $25.67,24.48$, $18.17,23.84,34.60$ and 36.69 per cent in apple, paddy, maize, vegetables, forest and pasture respectively.

The silt content varied from 20.10 to 49.50 , 28.89 to $47.80,43.56$ to $56.11,45.10$ to 50.20 , 22.10 to 53.60 and 20.10 to 50.02 per cent with the mean values of $41.19,37.34,49.16$, $48.20,39.24$ and 39.61 per cent in apple, paddy, maize, vegetables, forest and pasture respectively.

The clay fraction varied from 10.20 to 35.20 , 22.00 to $39.65,21.14$ to $35.60,10.50$ to 36.50 , 8.00 to 33.54 and 9.00 to 34.54 per cent with the mean values of $29.99,33.05,29.90,26.01$, 21.66 and 20.56 per cent in apple, paddy, maize, vegetables, forest and pasture respectively.

The soils were found to possess medium to moderately fine texture with translocation of clay and its deposition in the lower layers. Similar observations were earlier reported by Najar et al., (2009), Ramzan et al., (2014) and Bashir et al., (2016).

\section{Soil reaction $(\mathrm{pH})$}

The $\mathrm{pH}$ value (Table 3 ) in the surface soils of the selected locations under different land uses ranged from 6.02 to 6.62 with a mean of 6.35 in apple, 6.11 to 6.40 with a mean of 6.27 in paddy, 6.30 to 7.30 with a mean of 6.85 in maize, 6.00 to 6.60 with a mean of 6.25 in vegetable and 5.70 to 6.60 with a mean of 6.23 in forest and 5.80 to 6.60 with a mean of 6.26 in pasture soils respectively. The low $\mathrm{pH}$ observed in soils might be attributed to relatively higher rainfall which increases the leaching of salts and higher content of organic matter which brings down $\mathrm{pH}$ by releasing organic acids. The results are in agreement with the experimental findings of Bhat (2009), Najar et al., (2009), Pal et al., (2013), Sharma et al., (2013), Ramzan et al., (2014) and Bashir et al., (2016).

\section{Electrical conductivity}

The electrical conductivity of the soil samples ranged from 0.05 to $0.20 \mathrm{dSm}^{-1}$ with a mean $0.12 \mathrm{dSm}^{-1}$ (apple), 0.15 to $0.34 \mathrm{dSm}^{-1}$ with a mean of $0.22 \mathrm{dSm}^{-1}$ (paddy), 0.27 to 0.78 $\mathrm{dSm}^{-1}$ with a mean of $0.61 \mathrm{dSm}^{-1}$ (maize), 0.40 to $0.46 \mathrm{dSm}^{-1}$ with a mean of $0.42 \mathrm{dSm}^{-1}$ (vegetables), 0.07 to $0.25 \mathrm{dSm}^{-1}$ with a mean $0.18 \mathrm{dSm}^{-1}$ (forest) and 0.11 to $0.41 \mathrm{dSm}^{-1}$ with a mean of $0.25 \mathrm{dSm}^{-1}$ (pasture) respectively in the surface soils of selected sites under selected locations (Table 3). Electrical conductivity showing decreasing trend may be due to leaching of soluble salts. These results are in conformity with Thangasamy et al., (2004), Rao et al., (2008), Dhale and Prasad (2009), Najar et al., (2009), Sharma et al., (2013) and Bashir et al., (2016).

\section{Cation exchange capacity}

The data in Table 3 revealed that cation exchange capacity under selected land uses varied from 13.50 to $17.75 \mathrm{Cmol}_{\mathrm{c}} \mathrm{kg}^{-1}$ with a mean of $15.19 \mathrm{Cmol}_{\mathrm{c}} \mathrm{kg}^{-1}$ (apple), 11.10 to $13.96 \mathrm{Cmol}_{\mathrm{c}} \mathrm{kg}^{-1}$ with a mean of $12.51 \mathrm{Cmol}_{\mathrm{c}}$ $\mathrm{kg}^{-1}$ (paddy), 12.20 to $16.45 \mathrm{Cmol}_{\mathrm{c}} \mathrm{kg}^{-1}$ with a mean of $14.49 \mathrm{Cmol}_{\mathrm{c}} \mathrm{kg}^{-1}$ (maize), 10.55 to $14.00 \mathrm{Cmol}_{\mathrm{c}} \mathrm{kg}^{-1}$ with a mean of $12.17 \mathrm{Cmol}_{\mathrm{c}}$ $\mathrm{kg}^{-1}$ (vegetables), 10.51 to $12.78 \mathrm{Cmol}_{\mathrm{c}} \mathrm{kg}^{-1}$ with a mean of $11.75 \mathrm{Cmol}_{\mathrm{c}} \mathrm{kg}^{-1}$ (forest) and 11.56 to $17.65 \mathrm{Cmol}_{\mathrm{c}} \mathrm{kg}^{-1}$ with a mean of 15.37 $\mathrm{Cmol}_{\mathrm{c}} \mathrm{kg}^{-1}$ (pasture) respectively in the studied area with significant differences 
among the locations. The differences in the cation exchange capacity can be ascribed to the differences in the organic matter content and clay when the higher values of the cation exchange capacity can be assigned to the higher amount of organic matter in the surface soils. These findings corroborate with the results of Wani et al., (2010), Pal et al., (2013), Sharma et al., (2013), Ramzan et al., (2014) and Bashir et al., (2016).

Table.1 Location of the soil

\begin{tabular}{|c|c|c|c|c|c|}
\hline $\begin{array}{l}\text { Land use } \\
\text { system }\end{array}$ & $\begin{array}{l}\text { Sample } \\
\text { Code }\end{array}$ & Location & $\begin{array}{l}\text { Altitude } \\
\text { (mtrs) }\end{array}$ & Latitidue & Longtitude \\
\hline \multirow{9}{*}{ APPLE } & $\mathrm{L}_{1} \mathrm{~S}_{1}$ & Goripora & 1550 & $34^{0} 21^{\prime} 31.478^{\prime \prime} \mathrm{N}$ & $74^{0} 25^{\prime} 48.726^{\prime \prime} \mathrm{E}$ \\
\hline & $\mathrm{L}_{1} \mathrm{~S}_{2}$ & Janwara & 1547 & $34^{0} 20^{\prime} 29.254^{\prime \prime} \mathrm{N}$ & $74^{0} 29^{\prime} 28.219^{\prime \prime} \mathrm{E}$ \\
\hline & $\mathrm{L}_{1} \mathrm{~S}_{3}$ & Seelo & 1560 & $34^{0} 19^{\prime} 47.669^{\prime \prime} \mathrm{N}$ & $74^{0} 25^{\prime} 37.704^{\prime \prime} \mathrm{E}$ \\
\hline & $\mathrm{L}_{2} \mathrm{~S}_{1}$ & Tapper & 1552 & $34^{0} 11^{\prime} 55.447^{\prime \prime} \mathrm{N}$ & $74^{0} 30^{\prime} 45.808^{\prime \prime} \mathrm{E}$ \\
\hline & $\mathrm{L}_{2} \mathrm{~S}_{2}$ & Nehalpora & 1637 & $34^{0} 08^{\prime} 50.5211^{\prime \prime} \mathrm{N}$ & 74031'20.547'” \\
\hline & $\mathrm{L}_{2} \mathrm{~S}_{3}$ & Hanjiwera & 1548 & $34^{0} 08^{\prime} 31.288^{\prime \prime} \mathrm{N}$ & $74^{0} 35^{\prime} 05.287^{\prime \prime} \mathrm{E}$ \\
\hline & $\mathrm{L}_{3} \mathrm{~S}_{1}$ & singpora & 1561 & $34^{0} 08^{\prime} 37.766^{\prime \prime} \mathrm{N}$ & $74^{0} 35^{\prime} 48.865^{\prime \prime} \mathrm{E}$ \\
\hline & $\mathrm{L}_{3} \mathrm{~S}_{2}$ & Singpora & 1554 & $34^{0} 08^{\prime} 54.181 ” \mathrm{~N}$ & 74 $36^{0} 34.616^{\prime \prime} \mathrm{E}$ \\
\hline & $\mathrm{L}_{3} \mathrm{~S}_{3}$ & Singpora & 1549 & $34^{0} 09^{\prime} 03.540^{\prime \prime} \mathrm{N}$ & 74037'15.145'"E \\
\hline \multirow{9}{*}{ PADDY } & $\mathrm{L}_{1} \mathrm{~S}_{1}$ & Uri & 1420 & $34^{0} 04^{\prime} 27.256^{\prime \prime} \mathrm{N}$ & $74^{0} 03$ '28.426”'E \\
\hline & $\mathrm{L}_{1} \mathrm{~S}_{2}$ & Chehal & 1551 & $34^{0} 09^{\prime} 5.171^{\prime \prime N}$ & $74^{0} 12^{\prime} 31.831^{\prime \prime} \mathrm{E}$ \\
\hline & $\mathrm{L}_{1} \mathrm{~S}_{3}$ & Sheeri & 1565 & $34^{0} 10^{\prime} 54.3566^{\prime \prime} \mathrm{N}$ & $74^{0} 18^{\prime} 16.813 \mathrm{E}$ \\
\hline & $\mathrm{L}_{2} \mathrm{~S}_{1}$ & Mirgund & 1827 & $34^{0} 04^{\prime} 40.435^{\prime \prime} \mathrm{N}$ & $74^{0} 29^{\prime} 19.605$ 'E \\
\hline & $\mathrm{L}_{2} \mathrm{~S}_{2}$ & Kunzar & 1739 & $34^{0} 05^{\prime} 05.513^{\prime \prime} \mathrm{N}$ & $74^{0} 30^{\prime} 36.068^{\prime \prime} \mathrm{E}$ \\
\hline & $\mathrm{L}_{2} \mathrm{~S}_{3}$ & Wussan & 1665 & $34^{0} 05^{\prime} 17.318^{\prime \prime} \mathrm{N}$ & $74^{0} 32^{\prime} 07.592^{\prime \prime} \mathrm{E}$ \\
\hline & $\mathrm{L}_{3} \mathrm{~S}_{1}$ & Dawlatpora & 1565 & $34^{0} 12^{\prime} 52.429{ }^{\prime \prime} \mathrm{N}$ & $74^{0} 28^{\prime} 03.354^{\prime \prime} \mathrm{E}$ \\
\hline & $\mathrm{L}_{3} \mathrm{~S}_{2}$ & Saloosa & 1567 & $34^{0} 11^{\prime} 53.833^{\prime \prime} \mathrm{N}$ & $74^{0} 27^{\prime} 36.881^{\prime \prime} \mathrm{E}$ \\
\hline & $\mathrm{L}_{3} \mathrm{~S}_{3}$ & Wagoora & 1627 & $34^{0} 10^{\prime} 00.274^{\prime \prime} \mathrm{N}$ & $74^{0} 25^{\prime} 40.386^{\prime \prime} \mathrm{E}$ \\
\hline \multirow{9}{*}{ MAIZE } & $\mathrm{L}_{1} \mathrm{~S}_{1}$ & Gantmulla & 1590 & $34^{\circ} 10 ’ 34.59 ” \mathrm{~N}$ & $74^{0} 16^{\prime} 11.971$ 'Е \\
\hline & $\mathrm{L}_{1} \mathrm{~S}_{2}$ & Gantmulla & 1545 & $34^{0} 10^{\prime} 17.212^{\prime \prime} \mathrm{N}$ & $74^{0} 14^{\prime} 45.361^{\prime \prime} \mathrm{E}$ \\
\hline & $\mathrm{L}_{1} \mathrm{~S}_{3}$ & Gantmulla & 1630 & $34^{0} 10^{\prime} 25.408^{\prime \prime} \mathrm{N}$ & $74^{0} 14^{\prime} 54.160^{\prime \prime} \mathrm{E}$ \\
\hline & $\mathrm{L}_{2} \mathrm{~S}_{1}$ & Checki & 1558 & $34^{0} 07^{\prime} 11.341^{\prime \prime} \mathrm{N}$ & $74^{0} 40^{\prime} 33.824^{\prime \prime} \mathrm{E}$ \\
\hline & $\mathrm{L}_{2} \mathrm{~S}_{2}$ & Kawoosa & 1572 & $34^{0} 06^{\prime} 34.438^{\prime \prime} \mathrm{N}$ & $74^{0} 38^{\prime} 47.212^{\prime \prime} \mathrm{E}$ \\
\hline & $\mathrm{L}_{2} \mathrm{~S}_{3}$ & Kakarpora & 1551 & $34^{0} 08^{\prime} 15.985^{\prime \prime} \mathrm{N}$ & $74^{0} 39^{\prime} 28.820^{\prime \prime} \mathrm{E}$ \\
\hline & $\mathrm{L}_{3} \mathrm{~S}_{1}$ & Minichakh & 1768 & $34^{0} 11 ' 10.939$ " $\mathrm{N}$ & $74^{0} 21 ' 51.429{ }^{\prime \prime} \mathrm{E}$ \\
\hline & $\mathrm{L}_{3} \mathrm{~S}_{2}$ & Chandoosa & 1956 & $34^{0} 09^{\prime} 09.299$ 'N & $74^{0} 22{ }^{\prime} 30.827^{\prime \prime} \mathrm{E}$ \\
\hline & $\mathrm{L}_{3} \mathrm{~S}_{3}$ & muqam & 1644 & $34^{\circ} 13^{\prime} 29.700^{\prime \prime} \mathrm{N}$ & $74^{\circ} 24^{\prime} 34.00^{\prime \prime} \mathrm{E}$ \\
\hline
\end{tabular}


Contd..

\begin{tabular}{|c|c|c|c|c|c|}
\hline \multirow{9}{*}{ VEGETABLES } & $\mathbf{L}_{1} \mathbf{S}_{1}$ & Watrigam & 1607 & $34^{0} 19^{\prime} 18.866 " N$ & 74022'25.327'"E \\
\hline & $\mathrm{L}_{1} \mathrm{~S}_{2}$ & Dangiwacha & 1577 & $34^{0} 32^{\prime} 03.134^{\prime} \mathrm{N}$ & $74^{0} .36^{\prime} 91.648^{\prime \prime} \mathrm{E}$ \\
\hline & $\mathrm{L}_{1} \mathrm{~S}_{3}$ & Bahrampura & 1594 & $34^{0} 19^{\prime} 38.454^{\prime \prime} \mathrm{N}$ & $74^{0} 23^{\prime} 38.426^{\prime} \mathrm{E}$ \\
\hline & $\mathrm{L}_{2} \mathrm{~S}_{1}$ & Mamoosa & 1609 & $34^{0} 06^{\prime} 41.226^{\prime \prime} \mathrm{N}$ & 74032'17.254"'E \\
\hline & $\mathrm{L}_{2} \mathrm{~S}_{2}$ & Narbal & 1561 & $34^{0} 07^{\prime} 11.485^{\prime \prime} \mathrm{N}$ & $74^{0} 40^{\prime} 33.469^{\prime \prime} \mathrm{E}$ \\
\hline & $\mathrm{L}_{2} \mathrm{~S}_{3}$ & Pattan & 1641 & $34^{0} 08^{\prime} 50.032^{\prime \prime} \mathrm{N}$ & $74^{0} 31^{\prime} 20.504^{\prime \prime} \mathrm{E}$ \\
\hline & $\mathrm{L}_{3} \mathrm{~S}_{1}$ & jamia masjid & 1557 & $34^{0} 08^{\prime} 53.025^{\prime \prime} \mathrm{N}$ & 74036’34.226"'E \\
\hline & $\mathrm{L}_{3} \mathrm{~S}_{2}$ & $\begin{array}{c}\text { Nowpora } \\
\text { jageer }\end{array}$ & 1620 & $34^{0} 13^{\prime} 1.900 " \mathrm{~N}$ & $74^{0} 13^{\prime} 29.70^{\prime \prime} \mathrm{E}$ \\
\hline & $\mathrm{L}_{3} \mathrm{~S}_{3}$ & Singpora & 1553 & $34^{0} 09^{\prime} 03.395^{\prime \prime} \mathrm{N}$ & 74037'14.439'” \\
\hline \multirow{9}{*}{ FOREST } & $\mathrm{L}_{1} \mathrm{~S}_{1}$ & Ferozpora & 2219 & $34^{0} 03^{\prime} 50.500^{\prime \prime} \mathrm{N}$ & $74^{0} 24^{\prime} 59.130^{\prime \prime} \mathrm{E}$ \\
\hline & $\mathrm{L}_{1} \mathrm{~S}_{2}$ & Yaru & 1567 & $34^{0} 22^{\prime} 54.291^{\prime \prime} \mathrm{N}$ & $74^{0} 19^{\prime} 42.815^{\prime \prime} \mathrm{E}$ \\
\hline & $\mathrm{L}_{1} \mathrm{~S}_{3}$ & Gulmarg & 2630 & $34^{0} 03^{\prime} 37.728^{\prime \prime} \mathrm{N}$ & $74^{0} 23^{\prime} 13.414^{\prime \prime} \mathrm{E}$ \\
\hline & $\mathrm{L}_{2} \mathrm{~S}_{1}$ & Gulmarg & 2540 & $34^{0} 02^{\prime} 31.451^{\prime \prime} \mathrm{N}$ & $74^{0} 23^{\prime} 51.385^{\prime \prime} \mathrm{E}$ \\
\hline & $\mathrm{L}_{2} \mathrm{~S}_{2}$ & Shranz & 2007 & $34^{0} 05^{\prime} 38.629^{\prime \prime} \mathrm{N}$ & $74^{0} 22^{\prime} 43.750^{\prime \prime} \mathrm{E}$ \\
\hline & $\mathrm{L}_{2} \mathrm{~S}_{3}$ & Rajpora & 2017 & $34^{0} 08^{\prime} 20.544^{\prime \prime} \mathrm{N}$ & $74^{0} 21^{\prime} 45.522^{\prime \prime} \mathrm{E}$ \\
\hline & $\mathrm{L}_{3} \mathrm{~S}_{1}$ & Nowshehra & 1582 & $34^{0} 09^{\prime} 16.110^{\prime \prime} \mathrm{N}$ & $74^{0} 13^{\prime} 27.53^{\prime \prime} \mathrm{E}$ \\
\hline & $\mathrm{L}_{3} \mathrm{~S}_{2}$ & Gantmulla & 1583 & $34^{0} 10^{\prime} 31.845^{\prime \prime} \mathrm{N}$ & $74^{0} 15^{\prime} 20.326^{\prime \prime} \mathrm{E}$ \\
\hline & $\mathrm{L}_{3} \mathrm{~S}_{3}$ & Mohra & 1441 & $34^{0} 08^{\prime} 41.555^{\prime \prime} \mathrm{N}$ & 7409'14.284'” \\
\hline \multirow{9}{*}{ PASTURE } & $\mathrm{L}_{1} \mathrm{~S}_{1}$ & Botingo & 1553 & $34^{0} 21^{\prime} 50.373^{\prime \prime} \mathrm{N}$ & $74^{0} 29^{\prime} 47.826^{\prime \prime} \mathrm{E}$ \\
\hline & $\mathrm{L}_{1} \mathrm{~S}_{2}$ & Duroo & 1552 & $34^{0} 21^{\prime} 02.074^{\prime \prime} \mathrm{N}$ & $74^{0} 27^{\prime} 55.216^{\prime \prime} \mathrm{E}$ \\
\hline & $\mathrm{L}_{1} \mathrm{~S}_{3}$ & Brath & 1569 & $34^{0} 20^{\prime} 07.265^{\prime \prime} \mathrm{N}$ & $74^{0} 26^{\prime} 13.402^{\prime \prime} \mathrm{E}$ \\
\hline & $\mathrm{L}_{2} \mathrm{~S}_{1}$ & uri & 1360 & $34^{0} 04^{\prime} 24.069^{\prime \prime} \mathrm{N}$ & $74^{0} 04^{\prime} 00.894^{\prime \prime} \mathrm{E}$ \\
\hline & $\mathrm{L}_{2} \mathrm{~S}_{2}$ & Boniyar & 1477 & $34^{0} 08^{\prime} 24.197^{\prime \prime} \mathrm{N}$ & $74^{0} 10^{\prime} 13.572^{\prime \prime} \mathrm{E}$ \\
\hline & $\mathrm{L}_{2} \mathrm{~S}_{3}$ & Pichlan & 1571 & $34^{0} 09^{\prime} 51.892^{\prime} \mathrm{N}$ & $74^{0} 14^{\prime} 09.263^{\prime \prime} \mathrm{E}$ \\
\hline & $\mathrm{L}_{3} \mathrm{~S}_{1}$ & shontpathri & 2270 & $34^{0} 05^{\prime} 13.224^{\prime \prime} \mathrm{N}$ & $74^{0} 23^{\prime} 30.463^{\prime \prime} \mathrm{E}$ \\
\hline & $\mathrm{L}_{3} \mathrm{~S}_{2}$ & Gulmarg & 2609 & $34^{0} 03^{\prime} 24.517^{\prime \prime} \mathrm{N}$ & $74^{0} 23^{\prime} 13.408^{\prime \prime} \mathrm{E}$ \\
\hline & $\mathrm{L}_{3} \mathrm{~S}_{3}$ & Gulmarg & 2614 & $34^{0} 03^{\prime} 34.306^{\prime \prime} \mathrm{N}$ & $74^{0} 23^{\prime} 12.887^{\prime \prime} \mathrm{E}$ \\
\hline
\end{tabular}




\section{Int.J.Curr.Microbiol.App.Sci (2018) 7(12): 948-961}

Table.2 Particle size distribution of soils of District Baramulla under different Land use systems

\begin{tabular}{|c|c|c|c|c|c|c|}
\hline \multirow[b]{2}{*}{ Land use System } & \multicolumn{6}{|c|}{ Soil separates ( \%) } \\
\hline & Coarse sand & Fine sand & Total sand & Silt & Clay & Textural class \\
\hline \multicolumn{7}{|l|}{ APPLE } \\
\hline $\mathrm{L}_{1} \mathrm{~S}_{1}$ & 0.22 & 43.32 & 43.54 & 21.62 & 34.76 & Clay loam \\
\hline $\mathrm{L}_{1} \mathrm{~S}_{2}$ & 0.17 & 44.45 & 44.62 & 20.10 & 34.68 & Clay loam \\
\hline $\mathrm{L}_{1} \mathrm{~S}_{3}$ & 2.68 & 37.31 & 39.99 & 49.50 & 10.20 & Silt loam \\
\hline $\mathrm{L}_{2} \mathrm{~S}_{1}$ & 3.18 & 18.21 & 21.39 & 47.66 & 30.55 & Silt clay loam \\
\hline $\mathrm{L}_{2} \mathrm{~S}_{2}$ & 3.00 & 25.24 & 28.24 & 47.20 & 23.80 & Silt loam \\
\hline $\mathrm{L}_{2} \mathrm{~S}_{3}$ & 2.85 & 19.25 & 22.10 & 46.21 & 31.62 & Silt clay loam \\
\hline $\mathrm{L}_{3} \mathrm{~S}_{1}$ & 3.20 & 16.10 & 19.30 & 45.30 & 34.90 & Silt clay loam \\
\hline $\mathrm{L}_{3} \mathrm{~S}_{2}$ & 3.40 & 15.20 & 18.60 & 46.10 & 35.20 & Silt clay loam \\
\hline $\mathrm{L}_{3} \mathrm{~S}_{3}$ & 3.60 & 15.00 & 18.60 & 47.00 & 34.20 & Silt clay loam \\
\hline Mean & 2.48 & 25.67 & 28.49 & 41.19 & 29.99 & - \\
\hline \multicolumn{7}{|l|}{ PADDY } \\
\hline $\mathrm{L}_{1} \mathrm{~S}_{1}$ & 3.24 & 16.62 & 19.86 & 46.33 & 33.20 & Silt clay loam \\
\hline $\mathrm{L}_{1} \mathrm{~S}_{2}$ & 3.12 & 26.53 & 29.65 & 30.56 & 39.65 & Clay loam \\
\hline $\mathrm{L}_{1} \mathrm{~S}_{3}$ & 2.20 & 24.20 & 26.40 & 47.80 & 24.85 & Silt loam \\
\hline $\mathrm{L}_{2} \mathrm{~S}_{1}$ & 5.80 & 26.20 & 32.00 & 32.10 & 35.30 & Clay loam \\
\hline $\mathrm{L}_{2} \mathrm{~S}_{2}$ & 10.56 & 24.48 & 35.04 & 42.92 & 22.00 & Loam \\
\hline $\mathrm{L}_{2} \mathrm{~S}_{3}$ & 1.20 & 18.02 & 19.22 & 45.50 & 35.10 & Silt clay loam \\
\hline $\mathrm{L}_{3} \mathrm{~S}_{1}$ & 3.62 & 26.23 & 29.85 & 31.56 & 37.65 & clay loam \\
\hline $\mathrm{L}_{3} \mathrm{~S}_{2}$ & 3.76 & 25.85 & 21.61 & 30.42 & 38.42 & Clay loam \\
\hline $\mathrm{L}_{3} \mathrm{~S}_{3}$ & 4.87 & 32.21 & 37.08 & 28.89 & 33.11 & Clay loam \\
\hline Mean & 4.26 & 24.48 & 27.86 & 37.34 & 33.05 & - \\
\hline
\end{tabular}


Int.J.Curr.Microbiol.App.Sci (2018) 7(12): 948-961

Contd..

\begin{tabular}{|c|c|c|c|c|c|c|}
\hline Land use System & Coarse sand & Fine sand & Total sand & Silt & Clay & Textural class \\
\hline \multicolumn{7}{|l|}{ MAIZE } \\
\hline $\mathrm{L}_{1} \mathrm{~S}_{1}$ & 2.20 & 18.01 & 20.21 & 56.11 & 21.14 & Silt loam \\
\hline $\mathrm{L}_{1} \mathrm{~S}_{2}$ & 1.95 & 18.77 & 20.72 & 54.23 & 22.88 & Silt loam \\
\hline $\mathrm{L}_{1} \mathrm{~S}_{3}$ & 2.68 & 18.48 & 21.16 & 55.60 & 23.22 & Silt loam \\
\hline $\mathrm{L}_{2} \mathrm{~S}_{1}$ & 1.30 & 18.10 & 19.40 & 45.40 & 34.40 & Silt clay loam \\
\hline $\mathrm{L}_{2} \mathrm{~S}_{2}$ & 2.88 & 17.64 & 20.52 & 44.22 & 35.00 & Silt clay loam \\
\hline $\mathrm{L}_{2} \mathrm{~S}_{3}$ & 1.62 & 18.23 & 19.85 & 43.56 & 35.25 & Silt clay loam \\
\hline $\mathrm{L}_{3} \mathrm{~S}_{1}$ & 1.32 & 17.10 & 18.42 & 46.10 & 35.40 & Silt clay loam \\
\hline $\mathrm{L}_{3} \mathrm{~S}_{2}$ & 2.30 & 19.90 & 22.20 & 52.10 & 26.20 & Silt loam \\
\hline $\mathrm{L}_{3} \mathrm{~S}_{3}$ & 1.80 & 17.30 & 19.10 & 45.10 & 35.60 & Silt clay loam \\
\hline Mean & 2.01 & 18.17 & 20.18 & 49.16 & 29.90 & - \\
\hline \multicolumn{7}{|l|}{ VEGETABLES } \\
\hline $\mathrm{L}_{1} \mathrm{~S}_{1}$ & 1.92 & 26.91 & 28.82 & 49.20 & 20.98 & Silt loam \\
\hline $\mathrm{L}_{1} \mathrm{~S}_{2}$ & 2.01 & 25.41 & 27.42 & 50.20 & 22.10 & Silt loam \\
\hline $\mathrm{L}_{1} \mathrm{~S}_{3}$ & 2.00 & 26.80 & 28.80 & 48.60 & 21.60 & Silt loam \\
\hline $\mathrm{L}_{2} \mathrm{~S}_{1}$ & 1.68 & 29.71 & 31.39 & 49.50 & 19.02 & Silt loam \\
\hline $\mathrm{L}_{2} \mathrm{~S}_{2}$ & 1.56 & 19.20 & 20.76 & 45.10 & 33.30 & Silt Clay loam \\
\hline $\mathrm{L}_{2} \mathrm{~S}_{3}$ & 2.68 & 37.31 & 39.99 & 49.50 & 10.50 & Silt loam \\
\hline $\mathrm{L}_{3} \mathrm{~S}_{1}$ & 3.45 & 16.80 & 20.25 & 49.10 & 36.50 & Silt clay loam \\
\hline $\mathrm{L}_{3} \mathrm{~S}_{2}$ & 1.10 & 16.30 & 17.40 & 47.30 & 35.20 & Silt clay loam \\
\hline $\mathrm{L}_{3} \mathrm{~S}_{3}$ & 3.20 & 16.10 & 19.30 & 45.30 & 34.90 & Silt clay loam \\
\hline Mean & 2.18 & 23.84 & 26.01 & 48.20 & 26.01 & - \\
\hline
\end{tabular}




\section{Contd..}

\begin{tabular}{|c|c|c|c|c|c|c|}
\hline $\begin{array}{l}\text { Land use } \\
\text { System }\end{array}$ & Coarse sand & Fine sand & Total sand & Silt & Clay & Textural class \\
\hline \multicolumn{7}{|c|}{ FOREST } \\
\hline $\mathrm{L}_{1} \mathrm{~S}_{1}$ & 1.20 & 42.20 & 43.40 & 22.10 & 33.54 & Sandy clay loam \\
\hline $\mathrm{L}_{1} \mathrm{~S}_{2}$ & 2.60 & 24.20 & 26.80 & 45.80 & 21.43 & Silt loam \\
\hline $\mathrm{L}_{1} \mathrm{~S}_{3}$ & 3.50 & 54.50 & 58.00 & 32.00 & 8.00 & Sandy loam \\
\hline $\mathrm{L}_{2} \mathrm{~S}_{1}$ & 2.65 & 52.35 & 55.00 & 36.00 & 9.00 & Sandy loam \\
\hline $\mathrm{L}_{2} \mathrm{~S}_{2}$ & 6.30 & 30.50 & 36.80 & 34.10 & 28.30 & Clay loam \\
\hline $\mathrm{L}_{2} \mathrm{~S}_{3}$ & 5.23 & 31.44 & 36.67 & 33.14 & 29.44 & Clay loam \\
\hline $\mathrm{L}_{3} \mathrm{~S}_{1}$ & 2.00 & 27.22 & 29.22 & 48.32 & 20.46 & Silt loam \\
\hline $\mathrm{L}_{3} \mathrm{~S}_{2}$ & 1.58 & 20.68 & 22.26 & 53.60 & 23.98 & Silt loam \\
\hline $\mathrm{L}_{3} \mathrm{~S}_{3}$ & 2.50 & 28.32 & 30.82 & 48.12 & 20.78 & Silt loam \\
\hline Mean & 3.06 & 34.60 & 37.66 & 39.24 & 21.66 & - \\
\hline \multicolumn{7}{|l|}{ PASTURE } \\
\hline $\mathrm{L}_{1} \mathrm{~S}_{1}$ & 7.30 & 29.50 & 36.80 & 35.10 & 27.10 & Clay loam \\
\hline $\mathrm{L}_{1} \mathrm{~S}_{2}$ & 0.16 & 45.20 & 45.36 & 20.10 & 34.54 & Sandy clay loam \\
\hline $\mathrm{L}_{1} \mathrm{~S}_{3}$ & 2.00 & 22.00 & 24.00 & 45.20 & 30.80 & Clay loam \\
\hline $\mathrm{L}_{2} \mathrm{~S}_{1}$ & 2.20 & 27.62 & 29.82 & 50.02 & 18.20 & Silt loam \\
\hline $\mathrm{L}_{2} \mathrm{~S}_{2}$ & 1.80 & 26.38 & 28.18 & 47.52 & 22.88 & Silt Loam \\
\hline $\mathrm{L}_{2} \mathrm{~S}_{3}$ & 2.50 & 24.61 & 27.11 & 48.54 & 23.56 & Silt loam \\
\hline $\mathrm{L}_{3} \mathrm{~S}_{1}$ & 8.10 & 27.50 & 35.60 & 36.10 & 27.30 & Clay loam \\
\hline $\mathrm{L}_{3} \mathrm{~S}_{2}$ & 2.84 & 51.16 & 54.00 & 36.00 & 10.00 & Sandy loam \\
\hline $\mathrm{L}_{3} \mathrm{~S}_{3}$ & 3.11 & 51.89 & 55.00 & 37.00 & 9.00 & Sandy loam \\
\hline Mean & 2.78 & 36.69 & 39.47 & 39.61 & 20.56 & - \\
\hline
\end{tabular}


Table.3 Physico-chemical characteristics in soils of District Baramulla under different land use systems

\begin{tabular}{|c|c|c|c|c|c|}
\hline Land use system & $\mathrm{pH} \quad(\mathbf{1 : 2 . 5})$ & $\mathrm{EC}\left(\mathbf{d S m}^{-1}\right)$ & OC $(\%)$ & $\mathrm{CEC}\left(\mathbf{C m o l}_{\mathbf{c}} \mathbf{k g}^{-1}\right)$ & $\mathrm{CaCO}_{3} \quad(\%)$ \\
\hline \multicolumn{6}{|l|}{ APPLE } \\
\hline $\mathrm{L}_{1} \mathrm{~S}_{1}$ & 6.36 & 0.15 & 3.01 & 17.75 & 0.12 \\
\hline $\mathrm{L}_{1} \mathrm{~S}_{2}$ & 6.24 & 0.18 & 2.40 & 16.11 & 0.18 \\
\hline $\mathrm{L}_{1} \mathrm{~S}_{3}$ & 6.62 & 0.14 & 1.90 & 14.00 & 0.23 \\
\hline $\mathrm{L}_{2} \mathrm{~S}_{1}$ & 6.55 & 0.10 & 2.10 & 15.55 & 0.19 \\
\hline $\mathrm{L}_{2} \mathrm{~S}_{2}$ & 6.43 & 0.20 & 1.97 & 14.12 & 0.21 \\
\hline $\mathrm{L}_{2} \mathrm{~S}_{3}$ & 6.02 & 0.09 & 1.50 & 13.45 & 0.28 \\
\hline $\mathrm{L}_{3} \mathrm{~S}_{1}$ & 6.41 & 0.05 & 2.80 & 17.33 & 0.15 \\
\hline $\mathrm{L}_{3} \mathrm{~S}_{2}$ & 6.28 & 0.08 & 1.88 & 13.50 & 0.25 \\
\hline $\mathrm{L}_{3} \mathrm{~S}_{3}$ & 6.25 & 0.10 & 2.00 & 14.88 & 0.20 \\
\hline Mean & 6.35 & 0.12 & 2.17 & 15.19 & 0.20 \\
\hline $95 \%$ C.I & $6.21-6.49$ & 0.080 .16 & $1.8-2.54$ & $13.95-16.42$ & $0.16-0.23$ \\
\hline \multicolumn{6}{|l|}{ PADDY } \\
\hline $\mathrm{L}_{1} \mathrm{~S}_{1}$ & 6.40 & 0.15 & 2.72 & 13.64 & 0.10 \\
\hline $\mathrm{L}_{1} \mathrm{~S}_{2}$ & 6.36 & 0.34 & 1.25 & 12.12 & 0.18 \\
\hline $\mathrm{L}_{1} \mathrm{~S}_{3}$ & 6.25 & 0.21 & 2.01 & 12.75 & 0.15 \\
\hline $\mathrm{L}_{2} \mathrm{~S}_{1}$ & 6.22 & 0.20 & 2.81 & 13.96 & 0.10 \\
\hline $\mathrm{L}_{2} \mathrm{~S}_{2}$ & 6.11 & 0.18 & 1.08 & 11.54 & 0.25 \\
\hline $\mathrm{L}_{2} \mathrm{~S}_{3}$ & 6.31 & 0.28 & 1.70 & 11.99 & 0.20 \\
\hline $\mathrm{L}_{3} \mathrm{~S}_{1}$ & 6.28 & 0.20 & 1.05 & 11.10 & 0.28 \\
\hline $\mathrm{L}_{3} \mathrm{~S}_{2}$ & 6.31 & 0.21 & 1.50 & 12.33 & 0.16 \\
\hline $\mathrm{L}_{3} \mathrm{~S}_{3}$ & 6.21 & 0.25 & 2.20 & 13.22 & 0.15 \\
\hline Mean & 6.27 & 0.22 & 1.81 & 12.52 & 0.17 \\
\hline 95\% C.I & $6.20-6.34$ & $0.18-0.27$ & $1.30-2.33$ & $11.78-13.25$ & $0.13-0.22$ \\
\hline
\end{tabular}




\section{Contd..}

\begin{tabular}{|c|c|c|c|c|c|}
\hline Land use system & $\mathrm{pH} \quad(\mathbf{1 : 2 . 5 )}$ & $\mathrm{EC}\left(\mathbf{d S m}^{-1}\right)$ & OC $(\%)$ & $\mathrm{CEC}\left(\mathbf{C m o l}_{\mathbf{c}} \mathbf{k g}^{-1}\right)$ & $\mathrm{CaCO}_{3} \quad(\%)$ \\
\hline \multicolumn{6}{|l|}{ MAIZE } \\
\hline $\mathrm{L}_{1} \mathrm{~S}_{1}$ & 7.20 & 0.27 & 1.25 & 12.62 & 0.46 \\
\hline $\mathrm{L}_{1} \mathrm{~S}_{2}$ & 6.90 & 0.75 & 3.08 & 15.14 & 0.22 \\
\hline $\mathrm{L}_{1} \mathrm{~S}_{3}$ & 7.00 & 0.61 & 3.40 & 15.76 & 0.21 \\
\hline $\mathrm{L}_{2} \mathrm{~S}_{1}$ & 6.70 & 0.52 & 1.08 & 12.20 & 0.65 \\
\hline $\mathrm{L}_{2} \mathrm{~S}_{2}$ & 6.50 & 0.65 & 4.60 & 16.45 & 0.18 \\
\hline $\mathrm{L}_{2} \mathrm{~S}_{3}$ & 6.80 & 0.71 & 2.05 & 13.11 & 0.43 \\
\hline $\mathrm{L}_{3} \mathrm{~S}_{1}$ & 6.30 & 0.55 & 2.06 & 14.33 & 0.41 \\
\hline $\mathrm{L}_{3} \mathrm{~S}_{2}$ & 7.30 & 0.78 & 4.01 & 16.31 & 0.20 \\
\hline $\mathrm{L}_{3} \mathrm{~S}_{3}$ & 7.00 & 0.65 & 2.70 & 14.50 & 0.33 \\
\hline Mean & 6.85 & 0.61 & 2.69 & 14.49 & 0.34 \\
\hline 95\% C.I & $6.60-7.10$ & $0.49-0.72$ & $1.77-3.61$ & $13.28-15.70$ & $0.22-0.46$ \\
\hline \multicolumn{6}{|l|}{ VEGETABLES } \\
\hline $\mathrm{L}_{1} \mathrm{~S}_{1}$ & 6.00 & 0.46 & 2.80 & 12.22 & 0.26 \\
\hline $\mathrm{L}_{1} \mathrm{~S}_{2}$ & 6.20 & 0.43 & 3.81 & 13.11 & 0.22 \\
\hline $\mathrm{L}_{1} \mathrm{~S}_{3}$ & 6.50 & 0.41 & 2.15 & 10.67 & 0.35 \\
\hline $\mathrm{L}_{2} \mathrm{~S}_{1}$ & 6.10 & 0.40 & 3.05 & 12.72 & 0.25 \\
\hline $\mathrm{L}_{2} \mathrm{~S}_{2}$ & 6.30 & 0.42 & 2.01 & 10.55 & 0.38 \\
\hline $\mathrm{L}_{2} \mathrm{~S}_{3}$ & 6.60 & 0.41 & 2.68 & 12.21 & 0.28 \\
\hline $\mathrm{L}_{3} \mathrm{~S}_{1}$ & 6.30 & 0.45 & 2.56 & 11.23 & 0.30 \\
\hline $\mathrm{L}_{3} \mathrm{~S}_{2}$ & 6.00 & 0.42 & 3.20 & 12.85 & 0.24 \\
\hline $\mathrm{L}_{3} \mathrm{~S}_{3}$ & 6.30 & 0.40 & 4.52 & 14.00 & 0.20 \\
\hline Mean & 6.25 & 0.42 & 2.98 & 12.17 & 0.28 \\
\hline $95 \%$ C.I & $6.09-6.41$ & $0.40-0.44$ & $2.36-3.58$ & $11.28-13.06$ & $0.23-0.32$ \\
\hline
\end{tabular}




\section{Contd..}

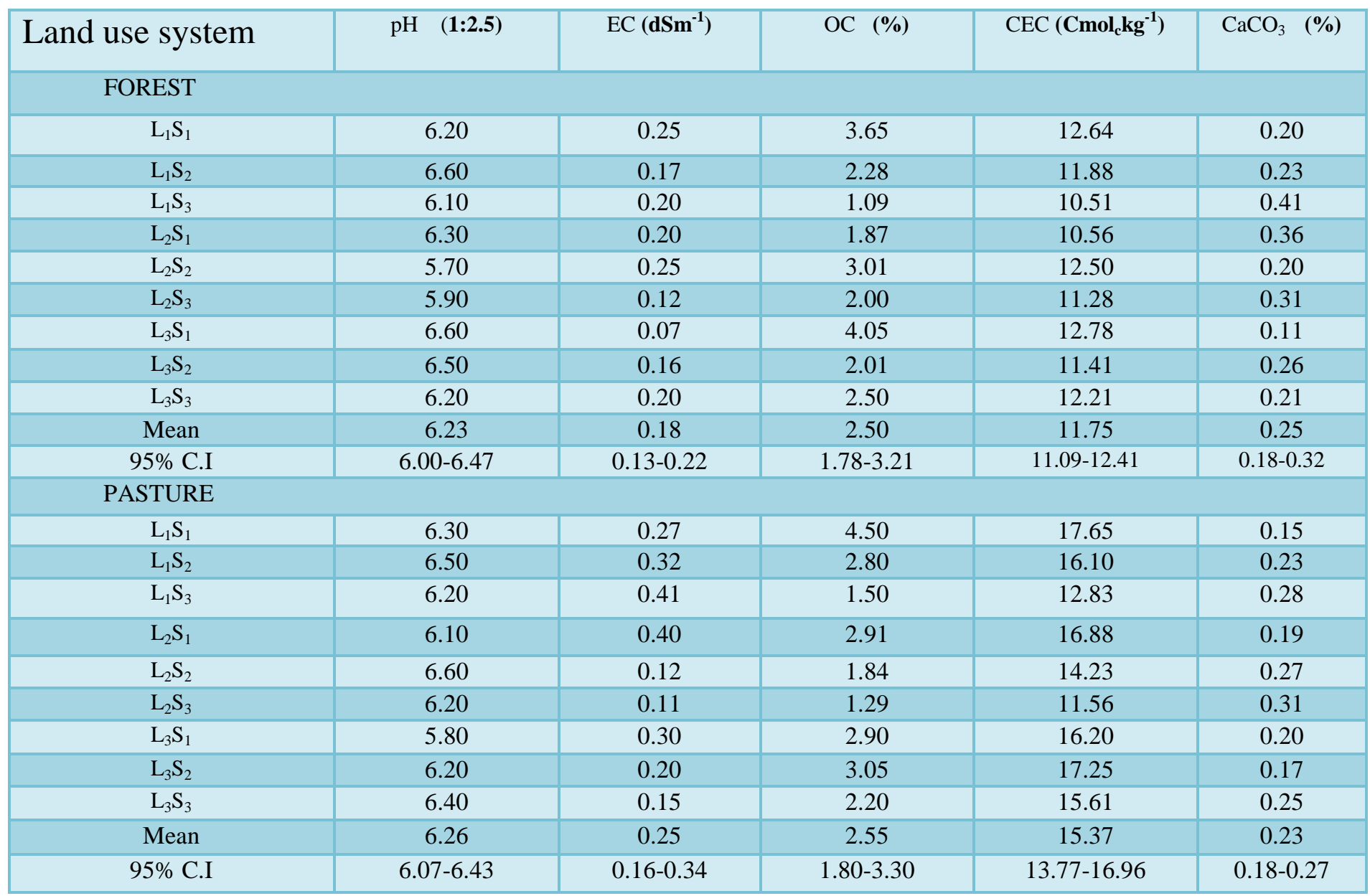


Fig.1

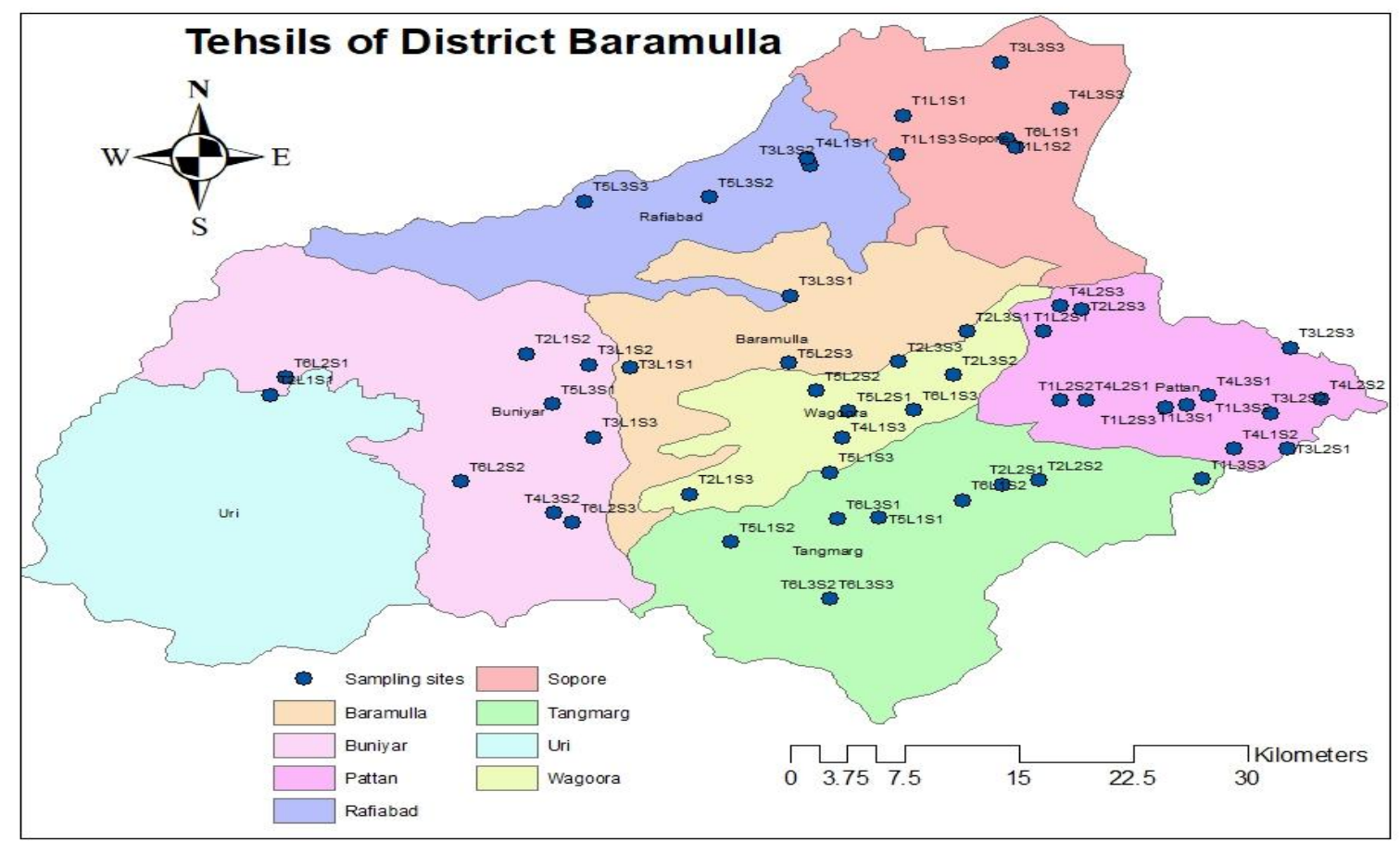




\section{Calcium carbonate}

The calcium carbonate content in surface soils under different land use systems varied from 0.12 to $0.28 \%$ with a mean of $0.20 \%$ (apple), 0.10 to $0.28 \%$ with a mean of $0.17 \%$ (paddy), 0.18 to $0.65 \%$ with a mean of $0.34 \%$ (maize), 0.20 to $0.38 \%$ with a mean of 0.28 $\%$ (vegetables), 0.11 to $0.41 \%$ with a mean of $0.25 \%$ (forest) and 0.15 to $0.31 \%$ with a mean of $0.23 \%$ (pasture) (Table 3). The low content of calcium carbonate can be attributed to the leaching down of calcium carbonate to the lower surfaces. These results are in conformity with the experimental findings of Kirmani (2004), Ramzan et al., (2014) and Bashir et al., (2016).

\section{Organic carbon}

The soils under study varied in the organic carbon content ranging between 1.50 to 3.01 per cent with a mean of 2.17 per cent (apple), 1.05 to 2.81 per cent with a mean of 1.81 per cent (paddy), 1.08 to 4.60 per cent with a mean of 2.69 per cent (maize), 2.01 to 4.52 per cent with a mean of 2.98 per cent (vegetables), 1.09 to 4.05 per cent with a mean of 2.50 per cent (forest) and 1.29 to 4.50 per cent with a mean of 2.55 per cent (pasture) under different locations (Table 3). The higher content of organic carbon may be due to incorporation of crop residues. The results are in conformity with the findings of (Palvu et al., 2007), Najar et al., (2009), Wani et al., (2010), Pal et al., (2013), Ramzan et al., (2014) and Bashir et al., (2016).

\section{References}

Abad, J. R. S., Khosravi, H. and Alamdarlou, E. H. 2014. Assessment the effects of land use changes on soil physicochemical properties in Jafarabad of Golestan province, Iran. Bulletin of Environment, Pharmacology and Life
Sciences; volume 3: 296-300.

Liu XL, He YQ, Zhang HL, Schroder JK, Li CL, Zhou J, Zhang ZY. Impact of land use and soil fertility on distributions of soil aggregate fractions and some nutrients. Pedosphere, 2010; 20(5):666673.

Bhat, S. N. 2009. Kinetics of potassium adsorption and desorption studies in Kashmir soils under different cropping sequences. M.Sc. dissertation submitted to Sher-e-Kashmir University of Agricultural Sciences and Technology of Kashmir, shalimar, pp 1-100.

Celik I. Land-use effects on organic matter and physical properties of soil in a southern Mediterranean highland of Turkey. Soil Tillage Research, 2005; 83:270-277.

Dhale, S.A. and Prasad, J. 2009. Characterization and classification of sweet orange growing soils of Jalna district, Maharashtra. Journal of the Indian Society of Soil Science 57(1): 1117.

Jackson, M. L. 1973. Soil Chemica Analysis. Prentice Hall of India, Private Limited, New Delhi: p 219-221.

Jackson, M. L. 1967. Soil Chemical Analysis. Prentice Hall of India, Private Limited, New Delhi: 498

Kirmani, N. A. 2004. Characterization, classification and development of Lacustrine soils of Kashmir valley. $P h$. D. thesis submitted to Sher-e-Kashmir University of Agricultural Sciences and Technology of Kashmir, Shalimar, pp. $1-96$.

Najar, G. R., Akhtar, F., Singh, S. R. and Wani, J. A. 2009. Characterization and classification of some apple growing soils of Kashmir. Journal of the Indian Society of Soil Science 57(1): 81-84.

Najar, G. A. 2002. Studies on Pedogenesis and nutrient indexing of apple (Red Delicious) growing soils of Kashmir. 
Thesis submitted to Sher-e-Kashmir University of Agricultural Sciences and Technology of Kashmir, Shalimar, pp. 1-204.

Pal, S., Panwar, P. and Bhardwaj, D. R. 2013. Soil quality under forest compared to other land-uses in acid soil of north western Himalaya, India. Annual Forest Research. 56(1): 187-198.

Pavlov, K. V. 2007. The assessment of the potassium status of soil by the proportion between different forms of potassium. Eurasian Soil Science. 40(7): 792-794.

Piper, C. 1966. Soil and Plant Analysis. Hans Publishers, Bombay.

Puri, A. N. 1930. A new method of estimating total carbonates in soils. Pusa Bulletin, No. 73, Imperial Agriculture Research, New Delhi.

Ramzan S, Bhat, M. A, Kirmani, N. A. and Rasool, R. (2014). Fractionation of Zinc and their Association with Soil Properties in Soils of Kashmir Himalayas. International Invention Journal of Agricultural and Soil Science, 0 Vol. 2(8): 132-142.

Rhoades, J. D. 1982. Cation exchange capacity. In: Methods of Soil Analysis: Chemical and Microbiological Properties. Part-II (Editors Page, A. L., Miller, R. H. and Keeney, D. R.). American Society of Agronomy and Soil Science Society of America, Madison, Wiscosin, USA.
Sharma, Y. K. 2013. Fertility status and potassium fractions of acid soils of Mokokchung, Nagaland under some important land use systems. Annuals of Plant and Soil Research. 15(2): 87-92.

Stutter, M. I., Deeks, L. K. and Billet, M. F. 2004. Spatial variability in soil exchange chemistry in agranitic upland catchment. Soil Science Society of American Journal. 68:1304-1314.

Thangasamy, A., Naidu, M. V. S. and Ramavatharam, N. 2004. Clay mineralogy of soils in the Sivagiri micro-waterhsed of Chittor District, Andhra Pradesh. Journal of the Indian Society of Soil Science; 52(4): 454-461.

Uzma Bashir, Tahir Ali and Fozia Qureshi. 2016. Distribution of different forms of potassium under temperature conditions of Kashmir. International Journal of Agriculture, Environment and Biotechnology. 9(2):213-219.

Walkley, A. and Black, C. A. 1934. An examination of Detjareff method for determining soil organic matter and a proposal modifications of the chromic acid titration method. Soil Science. 37: 29-38.

Wani, M. A., Mushtaq, Z. and Nazir, S. 2010. Mapping of micronutrients of the submerged rice soils of Kashmir. Research Journal of Agricultural Sciences, 1: 458-462.

\section{How to cite this article:}

Sumaira Shafi, Mushtaq A. Wani and Shaista Nazir. 2018. Assessment of Physico-chemical Properties in Some Soils of District Baramulla under Different Land Use Systems. Int.J.Curr.Microbiol.App.Sci. 7(12): 948-961. doi: https://doi.org/10.20546/ijcmas.2018.712.118 